\title{
PREVALÊNCIA DE ENTEROPARASITAS NA REGIÃO METROPOLITANA DE FORTALEZA, CEARÁ
}

\author{
Marcos Adelino ALMEIDA FILHO, Josiany Costa de SOUZA, Charles Ielpo MOURÃO \& Lydia Dayanne \\ Maia PANTOJA*
}

Universidade Estadual do Ceará. Fortaleza, Ceará, Brasil.

*Autor para correspondência: lydia.pantoja@uece.br

\section{RESUMO}

As enteroparasitoses são problemas de saúde pública associadas às condições socioeconômicas, principalmente pela falta de conhecimento de bons hábitos sanitários da população. Nesse ínterim, o presente trabalho objetivou a analisar prevalência de enteroparasitas em amostras fecais provenientes de pacientes da rede pública e privada da Região Metropolitana de Fortaleza - CE. A pesquisa se caracteriza em um estudo transversal, conduzido no período de abril/2014 a novembro/2016, com indivíduos provenientes da área metropolitana de Fortaleza, de ambos os sexos e diferentes faixas etárias. Do total de 6.596 amostras fecais analisadas de pacientes, 840 $(12,73 \%)$ apresentaram-se positivas para diversas formas parasitárias, prevalecendo os protozoários, as espécies Endolimax nana (55,14\%), Entamoeba coli $(21,33 \%)$ e Giardia lamblia $(13,95 \%)$ foram as mais frequentes. Seguido de nematelmintos como Ascaris lumbricoides (1,32\%), Trichocephalus trichiurus $(0,66 \%)$, Enterobius vermiculares $(0,33 \%)$ e os platelmintos foram Hymenolepis nana $(0,66 \%)$, Fasciola hepatica $(0,11 \%)$ e Taenia sp. $(0,11 \%)$. O número de casos positivos analisados nesta pesquisa, induz à necessidade da elaboração de políticas públicas, além de programas de educação e conscientização, principalmente para as populações carentes, com o intuito de amenizar a incidência e os impactos causados por enteroparasitoses.

Palavras-chave: Protozoário; Helminto; Fezes.

\begin{abstract}
Prevalence of parasitic diseases in the metropolitan region of Fortaleza in Ceará. Parasitic diseases are public health problems associated with socioeconomic conditions, mainly due to a lack of knowledge of good sanitary habits of a population. In this context, the present study aimed to analyze the prevalence of parasitic diseases in fecal samples from patients of the public and private network of the Metropolitan Region of Fortaleza - Ce. The research is characterized as a cross-sectional study, carried out from April 2014 to November 2016, with individuals from the metropolitan region of Fortaleza, of both sexes and different age groups. From the total of 6,596 fecal samples analyzed, $840(12.73 \%)$ were positive for several parasite forms, with the prevalence of protozoa, Endolimax nana (55.14\%), Entamoeba coli (21.33\%) and Giardia lamblia (13.95\%) were the most frequent. It was followed by nematelmines such as Ascaris lumbricoides (1.32\%), Trichocephalus trichiurus $(0.66 \%)$, Enterobius vermicularis $(0.33 \%)$ and flatworms were Hymenolepis nana (0.66\%), Fasciola hepatica (0.11\%) and Taenia sp. $(0.11 \%)$. The number of positive cases analyzed in this research leads to the need for public policies, as well as education and awareness programs, especially for the poor people in order to reduce the incidence and impacts caused by parasitic diseases.
\end{abstract}

Keywords: Protozoan; Helminth; Feces. 


\section{Biomedica Brasiliensia}

\section{Introdução}

As parasitoses intestinais são doenças causadas por agentes etiológicos protozoários ou helmintos, que são frequentemente encontrados nos seres humanos e que ainda representam relevante problema de saúde pública mundial (REY, 2008; NEVES et al., 2016). Estes, podem provocar variadas alterações patológicas e sua maior incidência pode estar relacionada a más condições situadas em regiões carentes, como no caso de países subdesenvolvidos. Dentre estas condições, destacam-se o saneamento básico ausente ou de baixa qualidade, falta de conscientização e de educação da sociedade, baixo desenvolvimento socioeconômico e descaso do poder público (BASSO et al., 2008; FREI; JUNCANSEN; PAES, 2008).

De acordo com a Organização Mundial da Saúde - OMS, as parasitoses intestinais representam as doenças mais comuns no mundo. Segundo a mesma, cerca de 1,4 bilhões de indivíduos encontram-se infectados por Ascaris lumbricoides, seguido com pouca diferença por Trichuris trichiura e pelos ancilostomídeos, 77 milhões por Taenia saginata, 10 milhões por Taenia solium e 70 milhões por Strongyloides stercoralis. Além disso, acredita-se também que 200 e 500 milhões de indivíduos, respectivamente, alberguem Giardia lamblia e Entamoeba histolytica (BAPTISTA et al., 2006; FONSECA; PEÑUELA; BOTERO, 2009; LODO et al., 2010; WHO, 2005).

No panorama mundial, os achados parasitológicos vêm sendo pouco estudados e pesquisados em decorrência da falta de estudos exploratórios desses dados. Tal situação não é diferente da encontrada no Brasil, assim como relata Ferraz et al. (2014), os estudos epidemiológicos são realizados de maneira fragmentada devido às dificuldades encontradas em organizar grandes inquéritos epidemiológicos que contemplem todas as características regionais e sociais envolvidas.

Ainda dentro do cenário nacional, essa deficiência de informações se torna preocupante em decorrência do perfil epidemiológico apresentado no país e em algumas das suas regiões, com destaque para o Norte e Nordeste, pois apresentam aumentos na disseminação dos agentes das parasitoses devido às condições socioeconômicas, principalmente pelo nível de instrução e falta de conhecimento de bons hábitos sanitários da população (BENINELO et al., 2011; FIRMOOLIVEIRA; AMOR, 2012).

Segundo Borges, Marciano e Oliveira (2011), os enteroparasitas mais frequentes no Brasil são os helmintos Ascaris lumbricoides, Trichuris trichiura e ancilostomídeos, os protozoários Entamoeba histolytica e Giardia lamblia, caracterizados por serem patogênicos, e os comensais Endolimax nana e Entamoeba coli.

$\mathrm{Na}$ região Nordeste os estudos divergem em relação aos parasitas prevalentes. No Maranhão, os achados positivos constataram a presença de Giardia lamblia, seguida de Ascaris lumbricoides (FERRAZ et al., 2014). Na Bahia e Rio Grande do Sul, a prevalência foi do parasita não patogênico Endolimax nana (SANTOS et al., 2013; SANCHES et al., 2014). Já em pesquisas realizadas em vários municípios da Região, como Independência (CE), Maracaçum (MA), Mucambo (CE), Santa Helena (MA), São José do Peixe (PI) e Ribeiro Gonçalves (PI), além de outras quatro cidades da Região Norte os achados se perfizeram em Ascaris lumbricoides mais presente, seguido de ancilostomídeos e Trichuris trichiura, respectivamente (FONSECA et al., 2010).

Segundo um estudo realizado no interior do Ceará, observou-se nas amostras a presença de enteroparasitas patogênicos e não patogênicos, sendo a Giardia prevalente na população analisada (14\%). Além deste gênero, outras formas parasitárias também encontradas foram: Enterobius vermicularis, Endolimax nana e Entomoeba coli (CALEGAR, 2015).

Diante do exposto, o presente trabalho objetivou a analisar prevalência de enteroparasitas em amostras fecais provenientes de pacientes da rede pública e privada da Região Metropolitana de Fortaleza - CE. 


\section{Biomedica Brasiliensia}

\section{Material e Métodos}

A presente pesquisa se caracteriza em um estudo transversal, cujo local de investigação se trata da Região Metropolitana de Fortaleza ( $3^{\circ} 43^{\prime} 6^{\prime \prime}$ Sul, 38 $32^{\prime} 36^{\prime \prime}$ Oeste), localizada no litoral norte, estado do Ceará, situado no Nordeste do Brasil, apresenta área territorial de $318,8 \mathrm{Km}^{2}$, um clima subtropical com temperaturas médias de $27^{\circ} \mathrm{C}$ e população estimada de 2.609 .716 habitantes (IBGE, 2016).

O estudo foi conduzido no período de abril de 2014 a novembro de 2016, com indivíduos provenientes da área metropolitana de Fortaleza, de ambos os sexos e diferentes faixas etárias, atendidos pela rede de Sistema Único de Saúde (SUS) e rede particular, que foram encaminhados a um laboratório prestador de serviço, para exames de rotina, não apresentando exclusivamente queixas relacionadas a parasitoses intestinais.

Os pacientes foram orientados a coletarem as fezes em coletor universal ou coletor comercial com conservante (de acordo com os protocolos operacionais do laboratório). Conforme solicitação médica os exames foram realizados em uma, duas ou três amostras para cada paciente.

Todas as amostras foram submetidas à técnica de sedimentação espontânea (Método de Hoffman, Pons e Janer) e/ou sedimentação por centrifugação (Método de Blagg) para pesquisa de cistos, ovos, larvas e oocistos. Para pacientes com suspeita de contaminação por Giardia lamblia ou Entamoeba sp., cujas fezes se apresentavam diarreicas, também era realizado exame direto e coloração por hematoxilina férrica (REY, 2008; NEVES et al., 2016).

Como requisitos éticos, foi resguardado o sigilo dos pacientes mantendo-se a identificação dos mesmos por um código numérico de uso exclusivo do laboratório, sendo disponibilizados apenas o código numérico, sexo e data de nascimento dos pacientes ora investigados. Para realização do levantamento dos dados, este estudo contou ainda com a autorização expressa e por escrito do responsável técnico do laboratório.

Os dados referentes à prevalência de enteroparasitas nas amostras fecais foram apresentados por meio de frequências simples e relativas e as discussões foram tratadas de acordo com a literatura disponível.

\section{Resultados e Discussão}

Do total de 6.596 amostras fecais analisadas de pacientes, $840(12,73 \%)$ apresentaram-se positivas para diversas formas parasitárias, com distribuição nos municípios de Fortaleza $(99,52 \%)$ e Maracanaú - CE (0,47\%) (figura 1). 


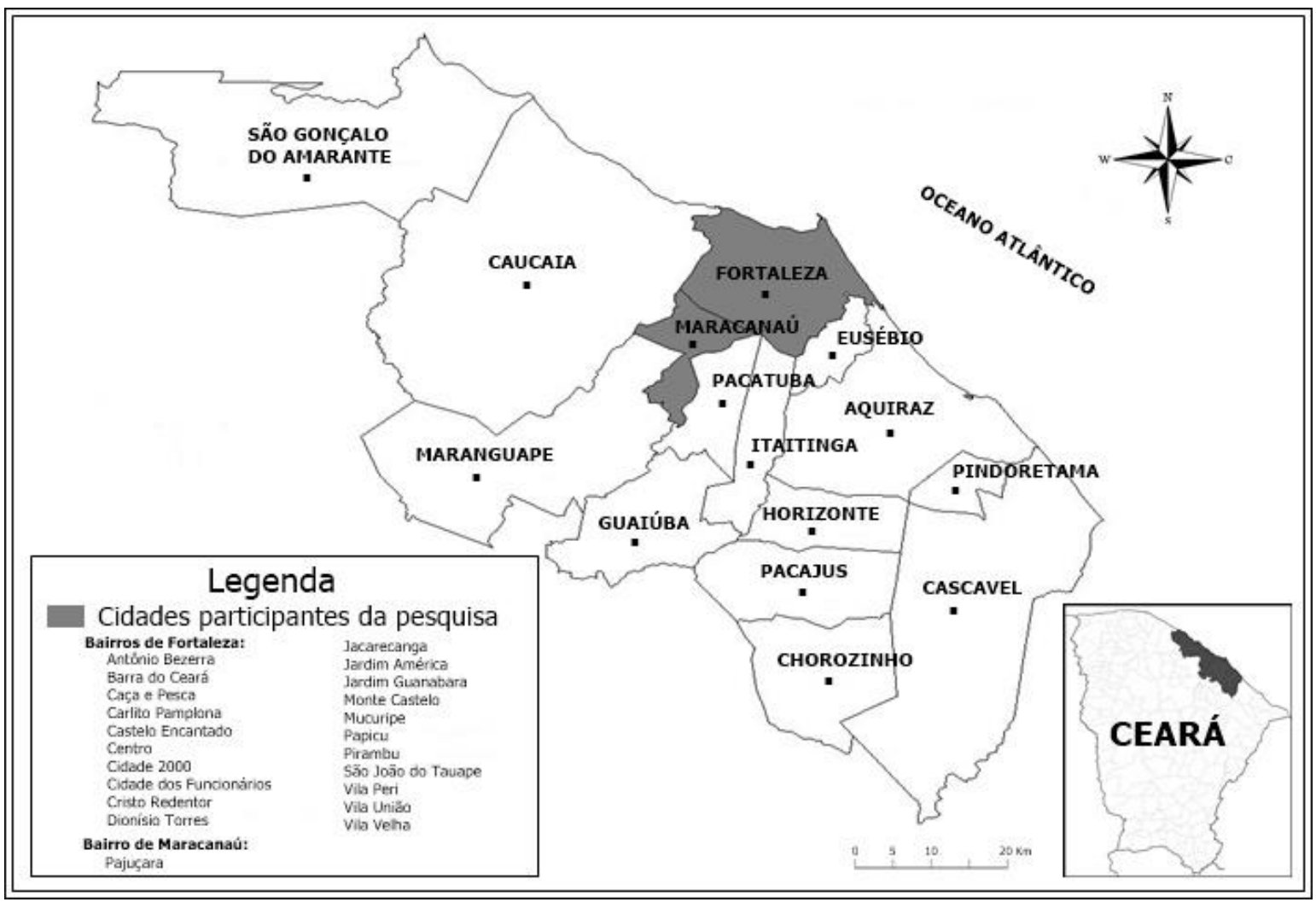

Figura 1: Localidades com amostras positivas para enteroparasitas em amostras fecais provenientes de pacientes da rede pública e privada da Região Metropolitana de Fortaleza - CE. Fonte: Adaptado do Instituto de Pesquisa e Estratégia Econômica do Ceará - IPECE.

Frente aos resultados positivos, $516(61,42 \%)$ pertencem ao sexo feminino e $324(38,57 \%)$ ao masculino. Estes dados se refletem em outras localidades do Brasil, em que é observada que a presença de casos é mais predominante no sexo feminino em relação ao masculino, na qual essa diferença pode chegar ao dobro das amostras positivas relatadas (MENEZES, 2013; MELO et al., 2015; ZANOTTO, 2015; ANTUNES et al., 2011). Em trabalho publicado, Gomes, Nascimento e Araujo (2007) relataram que a procura por serviços de saúde é diferenciada entre os sexos, na qual as mulheres culturalmente buscam o acesso ao atendimento básico e possuem um maior autocuidado em comparação aos homens.

No tocante a faixa etária (tabela 1), observou-se que de 0-11 anos, representada pelas crianças, uma maior prevalência de Endolimax nana $(11,29 \%)$, concordando com estudos realizados em crianças no interior do Ceará, na qual $24 \%$ de 75 exames positivos pertenciam a este parasita (SAMPAIO et al., 2013). Contudo, casos constantes relatados pela literatura, como exemplo no estudo de Silva et al. (2010), afirmam que o protozoário patogênico encontrado com maior frequência nessa faixa etária é a G. lamblia, mas, na presente pesquisa, o mesmo não corroborou com estes dados e teve ocorrência de $6,31 \%$.

Ainda na faixa etária de $0-11$ anos, $E$. coli $(3,43 \%)$ e E. histolytica $(0,44 \%)$ apresentaram menor prevalência quando comparado a resultados de outros estudos, na qual a ocorrência foi de $20 \%$ e $17 \%$ respectivamente (SAMPAIO et al., 2013). Nolla e Cantos (2005) afirmam que os protozoários comensais $E$. coli e E. nana podem gerar um agravo a saúde humana, sendo uma forma de contaminação é via oral e fecal, acabando por deixar as crianças com organismo mais expostos a outras parasitoses e patógenos.

Na faixa etária de 12-18 anos, representada pelos adolescentes, a maior prevalência dentre os casos positivos foi de E. nana (4,98\%), seguida por G. lamblia $(2,76 \%)$ e E. coli $(2,54 \%)$. Em estudo realizado na Bahia, cerca de $10,2 \%$ do público analisado encontrou-se positivo para 
parasitas na faixa etária semelhante (SAMPAIO et al., 2013). Santos e Merlini (2010) em sua pesquisa realizada com a população do município de Maria Helena, no Paraná, relataram 17,1\% de 69 casos positivos de enteroparasitoses na faixa etária de 10 a 19 anos, semelhante com a apresentada na presente pesquisa.

No grupo de 19-59 anos, que abrange os adultos, E. nana (27,02\%) prevaleceu, seguida por E. coli $(8,74 \%)$, G. lamblia $(3,10 \%)$, e E. histolytica $(2,10 \%)$. Já na faixa etária de 60 anos ou mais, representado pelos idosos, os endoparasitoses de maior ocorrência foram $E$. nana $(8,52 \%)$ e E. coli $(4,42 \%)$. Estes resultados corroboram com estudos realizados por Santos et al. (2013) na mesma faixa etária. A baixa porcentagem observada nesse grupo, provavelmente pode estar relacionada ao sistema imunológico de defesa adquirido ao longo dos anos, levando à redução das parasitoses (ZANOTTO, 2015). Outro fator seria uma maior consciência adquirida pelos adultos quanto às regras de higiene, surgidas no início do século passado, no qual a importância de se manter bons hábitos de higiene, como tomar banhos e escovar os dentes diariamente, e manter-se limpo, passou a ser divulgada e reforçada por cientistas e empresas de cosméticos, com o auxílio do avanço tecnológico (HIGIENE PESSOAL, 2017).

Os casos evidenciados na tabela 1 como "outros" são resultantes de exames não devidamente catalogados e que se encontravam com ausência de informação em relação à faixa etária. Neste grupo, assim como nas demais faixas etárias, observou-se a prevalência de $E$. nana $(3,32 \%)$ e E. coli $(2,21 \%)$.

Tabela 1: Distribuição e ocorrência de enteroparasitas por faixa etária de pacientes da rede pública e privada da Região Metropolitana de Fortaleza - CE.

\begin{tabular}{lccccc}
\hline \multirow{2}{*}{ Espécies } & \multicolumn{5}{c}{ Ocorrência (faixa etária) / no absoluto (\%) } \\
\cline { 2 - 5 } Endolimax sp. & $\mathbf{0 - 1 1}$ & $\mathbf{1 2 - 1 8}$ & $\mathbf{1 9 - 5 9}$ & $\mathbf{+ 6 0}$ & Outros \\
Endolimax nana & - & - & - & - & $1(0,11 \%)$ \\
Entamoeba coli & $102(11,29 \%)$ & $45(4,98 \%)$ & $244(27,02 \%)$ & $77(8,52 \%)$ & $30(3,32 \%)$ \\
Entamoeba histolytica & $31(3,43 \%)$ & $23(2,54 \%)$ & $79(8,74 \%)$ & $40(4,42 \%)$ & $20(2,21 \%)$ \\
Iodamoeba butschlii & $4(0,44 \%)$ & $7(0,77 \%)$ & $19(2,10 \%)$ & $8(0,88 \%)$ & $3(0,33 \%)$ \\
Giardia sp. & $1(0,11 \%)$ & $2(0,22 \%)$ & $6(0,66 \%)$ & $1(0,11 \%)$ & $1(0,11 \%)$ \\
Giardia lamblia & - & - & - & - & $1(0,11 \%)$ \\
Ancilostomideos & $57(6,31 \%)$ & $25(2,76 \%)$ & $28(3,10 \%)$ & $8(0,88 \%)$ & $8(0,88 \%)$ \\
Ancylostoma braziliense & - & - & - & - & $1(0,11 \%)$ \\
Ascaris lumbricoides & $3(0,33 \%)$ & $1(0,11 \%)$ & $5(0,55 \%)$ & - & $1(0,11 \%)$ \\
Enterobius vermiculares & $1(0,11 \%)$ & $1(0,11 \%)$ & - & - & $3(0,33 \%)$ \\
Trichuris trichiura & - & $1(0,11 \%)$ & - & - & $1(0,11 \%)$ \\
Trichocephalus trichiurus & $1(0,11 \%)$ & $2(0,22 \%)$ & $3(0,33 \%)$ & - & - \\
Fasciola hepatica & - & - & - & - & - \\
Hymenolepis nana & $1(0,11 \%)$ & $3(0,33 \%)$ & $1(0,11 \%)$ & - & $1(0,11 \%)$ \\
Taenia sp. & - & - & - & - & $1(0,11 \%)$ \\
\hline
\end{tabular}

Quanto aos enteroparasitas propriamente ditos (tabela 2), prevaleceram os protozoários, sendo as espécies Endolimax nana (55,14\%), Entamoeba coli (21,33\%) e Giardia lamblia $(13,95 \%)$ as mais frequentes. Os outros protozoários foram: Entamoeba histolytica $(4,54 \%)$, Iodamoeba butschlii $(1,21 \%)$, Endolimax sp. $(0,11 \%)$ e Giardia sp. $(0,11 \%)$.

Os nematelmintos foram Ascaris lumbricoides (1,32\%), Trichocephalus trichiurus $(0,66 \%)$, Enterobius vermiculares (0,33\%), Ancylostoma braziliense $(0,11 \%)$, Ancilostomideos 
$(0,11 \%)$ e Trichuris trichiura $(0,11 \%)$. Os platelmintos foram: Hymenolepis nana $(0,66 \%)$, Fasciola hepatica $(0,11 \%)$ e Taenia sp. $(0,11 \%)$.

Tabela 2. Ocorrência de enteroparasitas em amostras fecais provenientes de pacientes da rede pública e privada da Região Metropolitana de Fortaleza - CE.

\begin{tabular}{|c|c|c|c|c|}
\hline Grupo & Família & Espécies & Ocorrência & Porcentagem \\
\hline \multirow[t]{7}{*}{ Protozoário } & \multirow[t]{5}{*}{ Entamoebidae } & Endolimax sp. & 1 & $0,11 \%$ \\
\hline & & Endolimax nana & 498 & $55,14 \%$ \\
\hline & & Entamoeba coli & 193 & $21,37 \%$ \\
\hline & & Entamoeba histolytica & 41 & $4,54 \%$ \\
\hline & & Iodamoeba butschlii & 11 & $1,21 \%$ \\
\hline & \multirow[t]{2}{*}{ Hexamitidae } & Giardia sp. & 1 & $0,11 \%$ \\
\hline & & Giardia lamblia & 126 & $13,95 \%$ \\
\hline \multirow[t]{6}{*}{ Nematelminto } & \multirow[t]{2}{*}{ Ancylostomatidae } & Ancilostomideos & 1 & $0,11 \%$ \\
\hline & & Ancylostoma braziliense & 1 & $0,11 \%$ \\
\hline & Ascarididae & Ascaris lumbricoides & 12 & $1,32 \%$ \\
\hline & Oxyuridae & Enterobius vermicularis & 3 & $0,33 \%$ \\
\hline & \multirow[t]{2}{*}{ Trichuridae } & Trichuris trichiura & 1 & $0,11 \%$ \\
\hline & & Trichocephalus trichiurus & 6 & $0,66 \%$ \\
\hline \multirow[t]{4}{*}{ Platelminto } & Fasciolidae & Fasciola hepatica & 1 & $0,11 \%$ \\
\hline & Hymenolepididae & Hymenolepis nana & 6 & $0,66 \%$ \\
\hline & Taeniidae & Taenia sp. & 1 & $0,11 \%$ \\
\hline & \multicolumn{2}{|l|}{ Total } & 903 & \\
\hline
\end{tabular}

A frequência de enteroparasitoses observada neste estudo corrobora com trabalhos realizados em outros estados brasileiros. Santos et al. (2013) em seus estudos realizados no município de Santo Ângelo no Rio Grande do Sul, relataram a presença de 478 (19,3\%) casos positivos em 2470 exames analisados, na qual houve a prevalência de $E$. nana (36\%), seguida por E. coli (25\%), G. lamblia (16\%) e A. lumbricoides (5,6\%).

O parasita $E$. nana se destacou com dominância dentre as espécies encontradas, com um total de $498(55,14 \%)$ dos 840 resultados positivos, e com maior ocorrência em todas as faixas etárias. Este parasita, apesar de não ser patogênico, é comensal no intestino humano, mas que tem sido associado à diarreia infantil quando sua prevalência e intensidade é elevada (TOLEDO et al., 2009).

E. coli esteve presente em 193 (21,37\%) dos exames positivos. Assim como E. nana, este parasita é comensal, não é considerado patogênico, e pode ser encontrado colonizando o intestino humano, na qual esses parasitas utilizam a luz intestinal como abrigo e fonte de alimento, sem causar perturbações fisiológicas observáveis (CIMERMAN; CIMERMAN, 2010).

G. lamblia foi achado em $126(13,95 \%)$ dos casos positivos. Este é um protozoário patogênico, causador da giardíase que chega a atingir tanto o sexo masculino quanto o feminino, e tem prevalência comum em crianças (TAKIZAWA et al., 2008), assim como relatado na presente pesquisa. A giardíase, é uma infecção intestinal que pode ter relação com a precariedade das condições sanitárias e de tratamento de água, e alimentos contaminadas, que ao serem utilizados ou consumidos, o indivíduo estará ingerindo ou transmitindo os cistos infectantes desse parasita (CABRAL; ATWILL; BARBOSA, 2007).

Nas amostras analisadas, constataram-se diferentes casos de parasitismo (Tabela 3), sendo eles, com maior representatividade dos achados, o monoparasitismo $(92,97 \%)$, em maior presença 
no sexo feminino $(57,73 \%)$; biparasitismo $(6,54 \%)$, também com maior presença no sexo feminino $(3,57 \%)$; e poliparasitismo $(0,47 \%)$, com maior presença no sexo masculino $(0,35 \%)$. Nos casos de biparasitismo (tabela 4), as associações mais frequentes foram entre protozoários, com as espécies $E$. coli + E. nana ( 25 casos) e E. nana + G. lamblia ( 9 casos), essas relações têm sido observadas também em trabalhos realizados em outros estados do Brasil, como Amapá, Paraná Espírito Santo (MENEZES, 2013; ZANOTTO, 2015; LIMA et al., 2014).

Tabela 3. Casos de parasitismo em amostras fecais provenientes de pacientes da rede pública e privada da Região Metropolitana de Fortaleza - CE.

\begin{tabular}{lllll}
\hline \multirow{2}{*}{ Parasitismo } & \multicolumn{2}{c}{$\mathbf{N}^{\mathbf{0}}$ de casos } & \multicolumn{2}{c}{ Porcentagem (\%) } \\
\cline { 2 - 5 } & $\mathbf{M}$ & $\mathbf{F}$ & \multicolumn{1}{c}{$\mathbf{M}$} & $\mathbf{F}$ \\
\hline Monoparasitismo & 296 & 485 & $35,23 \%$ & $57,73 \%$ \\
Biparasitismo & 25 & 30 & $2,97 \%$ & $3,57 \%$ \\
Poliparasitismo & 3 & 1 & $0,35 \%$ & $0,11 \%$ \\
Total & \multicolumn{2}{c}{840} & & \\
\hline
\end{tabular}

Legenda: $\mathrm{M}$ - sexo masculino, $\mathrm{F}$ - sexo feminino.

Nos casos de poliparasitismo, 3 das 4 amostras as associações foram entre nematódeos protozoários (Tabela 4), com destaque para a espécie não patogênica E. coli. Estes resultados, apesar de serem baixos, representam dados importantes que podem ser possivelmente devido às baixas condições socioeconômicas e hábitos sanitários dos pacientes (BUSCHINI et al., 2007), que podem contribuir tanto para a presença do parasito, como também para a frequência no número desses indivíduos. Frei, Juncansen e Paes (2008) também relataram em seus estudos uma porcentagem baixa de poliparasitismo, com 1,4\% dos casos. Já Melo et al. (2015), encontraram nos seus 197 laudos positivos, 64 (32,5\%) poliparasitados.

Tabela 4. Números de associações parasitárias nos achados positivas de pacientes provenientes de pacientes da rede pública e privada da Região Metropolitana de Fortaleza - CE.

\begin{tabular}{lcc}
\hline \multirow{2}{*}{ Espécies } & \multicolumn{2}{c}{$\mathbf{N}^{\mathbf{0}}$ de casos } \\
\cline { 2 - 3 } & F & $\mathbf{M}$ \\
\hline A. lumbricoides + E. nana & - & 1 \\
E. nana + E. coli & 14 & 11 \\
E. nana + G. lamblia & 2 & 7 \\
E. coli + G. lamblia & 1 & 2 \\
E. coli + E. histolytica & - & 2 \\
E. nana + E. histolytica & 6 & 2 \\
E. nana + I. butschlii & 4 & - \\
E. coli + I. butschlii & 3 & - \\
A. lumbricoides + E. sp. + G. sp. & - & 1 \\
A. lumbricoides + E. nana + E. coli & - & 1 \\
G. lamblia + E. coli + A. lumbricoides & - & 1 \\
E. nana + E. coli + E. histolytica & 1 & - \\
\hline Legenda: - sexo masculino, F-sexo femino.
\end{tabular}

Legenda: $\mathrm{M}$ - sexo masculino, $\mathrm{F}$ - sexo feminino.

De acordo com os resultados deste trabalho, sugere-se que a população observada se encaixe no perfil analisado por outros autores na literatura do Brasil, na qual as más condições de vida da população influem diretamente e potencialmente na presença das enteroparasitoses e infecções intestinais que acometem as mais diversas faixas etárias. 


\section{Considerações Finais}

Na presente pesquisa, observou-se a prevalência de protozoários superior à de helmintos em todas as faixas etárias discutidas. A elevada presença dos parasitas não patogênicos E. nana e $E$. coli representou a maior prevalência dentre todas as espécies, em que ambos tiveram maior ocorrência em adultos (19 a 59 anos de idade).

G. lamblia representou a terceira maior prevalência neste estudo, dado que merece atenção por acometer principalmente crianças e estar relacionado principalmente às condições precárias de saneamento, tratamento de água e alimentos contaminados, situação observada na Região Metropolitana de Fortaleza-CE e em muitas localidades nacionais.

O número de casos positivos analisados nesta pesquisa, induz à necessidade da elaboração de políticas públicas, com metidas que estimulem hábitos higiênicos e sanitários adequados, além de programas de educação e conscientização, principalmente para as populações carentes, com o intuito de amenizar a incidência e os impactos causados por enteroparasitoses.

Nesse contexto vale ressaltar a importância de fazer estudos que realizem esse tipo de levantamento das parasitoses nos estados e em seus municípios, uma vez que há a escassez de dados que demonstrem a atual situação das parasitoses em brasileiros.

\section{Referências}

ANTUNES, R. M.; ANTUNES, J. V. M.; OLIVEIRA, L. G. A.; BELINELO, V. J.; VIEIRAFILHO, S. A. Prevalência de Enteroparasitoses em crianças de um centro escolar de ambiente rural de São Mateus, ES, Brasil. Enciclopédia Biosfera, v. 7, n. 12, p. 1-8, 2011.

BAPTISTA, S. C.; BREGUEZ, J. M. M.; BAPTISTA, M. C. P.; SILVA, G. M. S.; PINHEIRO, R. O. Análise da incidência de parasitoses intestinais no município de Paraíba do Sul, RJ. Rev. Bras. An. Clín., v. 38, n. 4, p. 271-273, 2006.

BASSO, R. M.; SILVA-RIBEIRO, R. T.; SOLIGO, D. S.; RIBACKI, S. I.; CALLEGARIJAQCUES, S.; ZOPPAS, B. C. Evolução da prevalência de parasitoses intestinais em escolares em Caxias do Sul, RS. Rev. Soc. Bras. Med. Trop., v. 41, p. 263-268, 2008.

BENINELO, V. G.; MILANEZES, B. A.; ROSA, A. B1.; BUSSULAR, J. P. B.; MORAES, A. N.; VIEIRA-FILHO, S. A. Intestinal parasites in students 10 to 15 years of school of São Matheus`s periphery, ES. Brazil. Eur. J. Scie. Res., v. 53, n. 2, p. 171-178, 2011.

BORGES, Q. F.; MARCIANO, F. M.; OLIVEIRA, H. B. Parasitos intestinais: elevada prevalência de Giardia lamblia em pacientes atendidos pelo serviço público de saúde da região sudeste de Goiás, Brasil. Rev. Patolog. Tropic., v. 40, n. 2, p. 149-157, 2011.

BUSCHINI, M. T.; PITTNER, E.; CZERVINSKI, T.; MORAES, I. F.; MOREIRA, M. M.; SANCHES, H. F. Spatial distribution of enteroparasites among school children from Guarapuava, State of Paraná, Brazil. Rev. Bras. Epidemiol., v. 10. n. 4, p. 568-78, 2007.

CABRAL, M. G. P.; ATWILL, E. R.; BARBOSA, A. P. Prevalência e fatores de riscos associados à infecção por Giardia lamblia em crianças hospitalizadas com diarreia em Goiânia, Goiás, Brasil. Rev. Inst. Med. Trop., v. 49, n. 3, p. 139-145, 2007. 
CALEGAR, D. A. Parasitismo intestinal e fatores associados em quatro localidades rurais no semiárido nordestino brasileiro [dissertação]. Pós-graduação em Medicina Tropical. Instituto Oswaldo Cruz. Rio de Janeiro; 2015.

CIMERMAN B, CIMERMAN S. Parasitologia humana e seus fundamentos gerais. 2 ed. São Paulo: Atheneu; 2010.

FERRAZ, R. R. N.; BARNABÉ, A. S.; PORCY, C.; JÚNIOR, A. D.; FEITOSA, T.; FIGUEIREDO, P. M. Parasitoses intestinais e baixos índices de Gini em Macapá (AP) e Timon (MA), Brasil. Cad. Saúde Colet., v. 22, n. 2, p. 173-176, 2014.

FIRMO-OLIVEIRA, V.; AMOR, A. L. M. Associação entre ocorrência de parasitas intestinais e diferentes variáveis clínicas epidemiológicas em moradores da comunidade Ribeiro, Araci, Bahia, Brasil. RBAC., v. 44, n. 1, p. 15-25, 2012.

FONSECA, E. O. L.; TEIXEIRA, M. G.; BARRETO, M. L.; CARMO, E. H.; COSTA, M. C. N. Prevalência e fatores associados às geo-helmintíases em crianças residentes em municípios com baixo IDH no Norte e Nordeste brasileiros. Cad. Saúde Pública, v. 26, n. 1, p. 143-152, 2010.

FONSECA, J. C.; PEÑUELA, R. M.; BOTERO, A. M. Parasitosis intestinal em niños de zonas palúdicas de Antioquia (Colombia). Iatreia, v. 22, n. 1, p. 27-46, 2009.

FREI, F.; JUNCANSEN, C.; PAES, J. T. Levantamento epidemiológico das parasitoses intestinais: viés analítico decorrente do tratamento profilático. Cad. Saúde Pública, v. 24, n. 12, p. 2919-1925, 2008.

GOMES, R.; NASCIMENTO, E. F.; ARAUJO, F. C. Por que os homens buscam menos os serviços de saúde do que as mulheres? As explicações de homens com baixa escolaridade e homens com ensino superior. Cad. Saúde Pública, v. 23, n. 1, p. 565-574, 2007.

HIGIENE PESSOAL, 2017. Higiene pessoal. Disponível em: < http://higiene-pessoal.info/>. Acesso em: 9 mar. 2017.

IBGE, 2016. Ceará, Fortaleza. Disponível em: < http://www.cidades.ibge.gov.br/xtras/perfil.php?lang=\&codmun=230440>. Acesso em: 26 jan 2017.

LIMA, M. S.; DAMÁZIO, S. M.; SOARES, A. R.; PRADO, G. P.; SOUZA, M. A. A. Intestinal parasites in children enrolled in early childhood centers of São Mateus, state of Espírito Santo, Brazil. Rev. Ciênc. Méd. Biol., v. 13, n. 2, p. 147-151, 2014.

LODO, M.; OLIVEIRA, C. G. B.; FONSECA, A. L. A.; CAPUTTO, L. Z.; PACKER, M. L. T.; VALENTI, V. E.; FONSECA, F. L. A. Prevalência de endoparasitas em município do interior paulista. Rev. Bras. Cresc. e Desenvolv. Humano, v. 20, n. 3, 769-777, 2010.

MELO, A. R.; ERICEIRA, F. V.; OLIVEIRA, N. D.; ROCHA, J. R.; FIRMO, W. C. A. Ocorrência de parasitos intestinais em laudos parasitológicos de fezes de um laboratório privado no município de Bacabal-MA. Enciclopédia Biosfera, v. 11, n. 21, p. 3420-30, 2015. 
MENEZES, R. A. O. Caracterização epidemiológica das enteroparasitoses evidenciadas na população atendida na Unidade Básica de Saúde Congós no município de Macapá - Amapá [dissertação]. Mestrado em Ciências da Saúde - Programa de Pós-Graduação em Ciências da Saúde, Departamento de Pós-Graduação, UNIFAP, Amapá; 2013.

NEVES, D. P.; MELO, A. L. DE; LINARDI, P. M.; VITOR, R. W. A. Parasitologia Humana. 13. ed. Rio de Janeiro: Atheneu; 2016.

NOLlA, A. C.; CANTOS, G. A. Relação entre a ocorrência de enteroparasitoses em manipuladores de alimentos e aspectos epidemiológicos em Florianópolis, Santa Catarina, Brasil. Cad. Saúde Pública, v. 21, n. 2, p. 641-645, 2005.

REY, L. Parasitologia. 4. ed. Rio de Janeiro: Ed. Guanabara Koogan; 2008.

SAMPAIO, M. G. V.; ALMEIDA, B. S.; SILVA, M. J.; SANTOS, B. S.; OLIVEIRA, A. D. L. Investigação de Enteroparasitoses em Pré-Escolares no Município de Juazeiro do Norte, Ceará. I CONIBIO/ II CONABIO/ VI SIMCBIO (v.2). Universidade Católica de Pernambuco Recife - PE; 2013.

SANTOS, C. S.; SOUZA, P. S. A.; FRIZZO, M. N.; MALlET, E. K. V.; PEDROSO, D. Prevalência de Enteroparasitoses e sua relação com Eosinofilia e Anemia em pacientes no município de Santo Ângelo, Rio Grande do Sul. Brasil. Rev. Saúde Integrada, v. 6, n. 1, p. 11$12,2013$.

SANTOS, S. A.; MERLINI, L. S. Prevalência de enteroparasitoses na população do município de Maria Helena, Paraná. Ciênc. Saúde Coletiva, v. 15, n. 3, p. 899-905, 2010.

SANCHES, F. G.; MACHADO, E. C. S.; SOARES, R. P.; NORBERG, A. N.; OLIVEIRA, J. T. M.; SERRA-FREIRE, N. M. Parasitismo Intestinal na Comunidade Rural de Marancó, Município de Santa Brígida, Estado da Bahia, Brasil. Rev. Saúde Física \& Mental, v. 3, n. 2, p. 39-49, 2014.

SILVA, F. S.; PAUlO, A. D. C.; BRAGA, C. M. M.; AlMEIDA, R. J.; GALVÃO, V. P. Frequência de parasitos intestinais no Município de Chapadinha, Maranhão, Brasil. Rev. Patologia Trop., v. 39, n. 1, p. 63-68, 2010.

TAKIZAWA, M. G. M. H.; SILVAR, L. L.; CELINSKINKI, B. F.; LIBERALI, G.; GANASSIN, L.; PROKOSKI, K.; MENEZES, V. C. Ocorrência de giardíase em crianças de duas creches do Município de Cascavel, Paraná, Brasil. Rev. Bras. Bioc., v. 63, n. 6, 2008.

TOLEDO, M. J. O.; PALUDETTO, A. W.; MOURA, F. T.; NASCIMENTO, E. S.; CHAVES, M.; ARAÚJO, S. M.; MOTA, L. T. Evaluation of enteroparasite control activities in a Kaingáng community of Southern Brazil. Rev. Saúde Pública, v. 43, n. 6, p. 981-990, 2009.

WHO. World Health Organization. Worldwide prevalence of anaemia 1993-2005. WHO Global Database on Anaemia, 2005.

ZANOTTO J. Ocorrência de Parasitoses Intestinais em Pacientes Atendidos em Laboratório Privado da Cidade de Cascavel - Paraná [monografia]. Conclusão de curso - Curso de Bacharelado em Farmácia, Faculdade Assis Gurgacz, Cascavel; 2015. 